\title{
"I Answered Obediently to 'She." "' Experiencing Pregnancy as a Nonbinary Person in the United States
}

Cody Miller Pyke, JD, LLM

The National Perinatal Association (NPA)is an interdisciplinary organization that strives to be a leading voice for perinatal care in the United States. Our diverse membership is comprised of healthcare providers, parents \& caregivers, educators, and service providers, all driven by their desire to give voice to and support babies and families at risk across the country.

Members of the NPA write a regular peer-reviewed column in Neonatology Today.

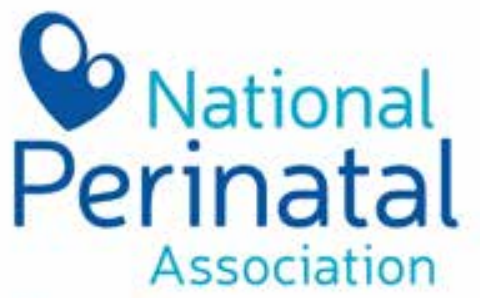

Educate. Advocate. Integrate.

"This year the chosen theme focused on the 4th Trimester and the various areas of vulnerability for parents and infants during this time, as well as the resources available for support."

\section{Readers can also follow}

via our Twitter Feed
In Summer 2020, Morgan McCarter(*) discovered something incredible. They were pregnant. Not "they," as in "Morgan and Morgan's partner," but "they"- the singular third-person pronoun(1) for Morgan, a non-binary person living in the United States. Morgan was both delighted and concerned. The pregnancy was planned, but they worried about the emotional toll of what they already suspected would be a highly gendered experience. Their anxiety was further deepened because they are not fully 'out' to the world or their biological family as a non-binary person. "I am not open to my family, nor am I really in a place emotionally to explain [my gender] to others. Basically, I am only openly myself in queer spaces or with those few people that I know are safe to be open with."

Transgender, non-binary, and genderexpansive (TGE) people are not novel, nor are they a rarity. Estimates report that 1 in 6 members of Generation $Z$ identify as LGBTQ+,(2) and this generation is already well into reproductive age.(3) For many TGE individuals, the experience of being misgendered-being referred to by pronouns or gendered terms which conflict with one's gender identity-is traumatic and contributes to psychological distress. (4) Despite the known harmful effects of misgendering TGE patients, multiple studies have shown that medical education on transgender health is desperately lacking in the United States and Canada.(5) Worse still, one study reported that " $26 \%$ of transgender individuals experienced discrimination in the health care system due to their gender identity and $52 \%$ were unable to obtain health service due to financial issues."(6 )

Morgan was fortunate to have health insurance and access to healthcare. However, what should have been an affirming prenatal experience of their first pregnancy was overshadowed by family and health professionals misgendering them and propagating unnecessarily gendered stereotypes.

"Birth classes were very gendered...[Everyone's] obsession with the baby's 'gender,' even when I decided not to find out the sex until birth... 'choose blue for boy or pink for girl!' It is difficult to face the dissonance of wanting to appreciate your family for their love, [but also] feel unheard when you say things like 'It's sex, not gender,' 'gender doesn't have a color' [and] 'my child will be able to wear what they want to."'

The dissonance was not only external. Like many TGE individuals who become pregnant, the physiology of pregnancy also triggered Morgan's gender dysphoria. "I struggled a lot with both the physical and emotional aspects of pregnancy. [As my body changed,] I had many small breakdowns about what I can only describe as 'body horror' [which] I needed to face [because of both my own intrinsic feelings and the extrinsic reactions [to my body] of the society around me. It was lucky that [because of the pandemic] I wasn't seeing very many people anyways."

Morgan's interactions with health professionals during labor and delivery were also mixed. Speaking of the staff at the birth center where delivery was to occur, Morgan described their midwife as "trauma-informed and also respectful of their pronouns," and their doula as "also very respectful." Unfortunately, at nearly 2 feet in length and more than 10 pounds, Morgan's baby was large-especially for a primigravid parent. After an induced trial of labor at the birth center, Morgan was ultimately transferred to a hospital for a Cesarean section.

By the time they arrived at the hospital, Morgan described themselves as "desperate" and that this desperation "overrode" their concerns about their own body and what effects surgical interventions may have on their dysphoria. "I did not bring up my gender identity [in the hospital]. I answered obediently to 'she' and 'her' and terms like 'mother.' I even referred to myself as such." Morgan felt "bizarre" and now only had their partner and the birth center doula who knew their gender identity. The hospital staff was professional and compassionate, but none stopped to ask about Morgan's gender identity, pronouns, or their history of trauma. All simply assumed they were a self-identifying woman with no reservations of how their body was to be discussed and treated. "The worst were the lactation specialists pushing for breastfeeding. The pressure to make me breastfeed made me feel sick because I was still unsure of [how I felt about breastfeeding]. One [lactation specialist] pushed me so hard. She told me that it should hurt and that she didn't care that my nipples were scabbing over."

Despite taking home a healthy newborn, 
Morgan's troubles continued at home. "My relationship with breastfeeding still feels a bit abusive even now. People in my life take breastfeeding for granted and don't understand my struggles with breastfeeding-even my partner who knows about my dysphoria. It is all just a continuation of the assigned-female-at-birth experience of my life... [where I'm unable] to assert autonomy over my body and mind. [Being treated this way] was one of the main reasons why I was staunchly against having children for a long time."

To date, Morgan and their partner are doing well and absolutely in love with their new baby. Nonetheless, when asked what they wished more health professionals knew about non-binary gestational parents, Morgan had this to say: "Just, everything. Be informed. Be aware. Have pronouns and gender identity be a part of the paperwork-and give more options than just male/female. People don't want to have to take extra steps to explain or justify themselves."

\section{References:}

1. For a primer on the grammatical correctness in English of singular "they," see https://en.wikipedia.org/wiki/Singular they.

2. https://www. washingtonpost.com/dc-md-va/2021/02/24/ gen-z-labt/

3. https://www.pewresearch.org/fact-tank/2019/01/17/wheremillennials-end-and-generation-z-begins/

4. Cooper, K., Russell, A., Mandy, W., \& Butler, C. (2020). The phenomenology of gender dysphoria in adults: A systematic review and meta-synthesis. Clinical psychology review, 80, 101875. https://doi.org/10.1016/j.cpr.2020.101875

5. Korpaisarn, S., \& Safer, J. D. (2018). Gaps in transgender medical education among healthcare providers: A major barrier to care for transgender persons. Reviews in endocrine \& metabolic disorders, 19(3), 271-275.

6. Kenagy G. P. (2005). Transgender health: findings from two needs assessment studies in Philadelphia. Health \& social work, 30(1), 19-26.

(*) The patient interviewee's name has been changed for privacy reasons. Details on the birth are also kept appropriately vague for privacy.

Disclosure: The National Perinatal Association www.nationalperinatal.org is a $501 \mathrm{c} 3$ organization that provides education and advocacy around issues affecting the health of mothers, babies, and families.

\section{NT}

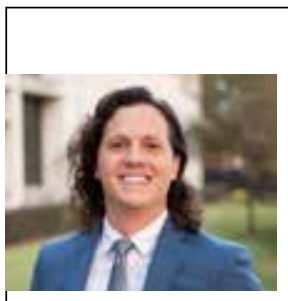

Corresponding Author

Cody Miller Pyke, JD, LLM, MSBE,

MD Candidate Class of 2022

Baylor Colllege of Medicine

Houstoh, Texas

Email: :cody.miller@nationalperinatal.org
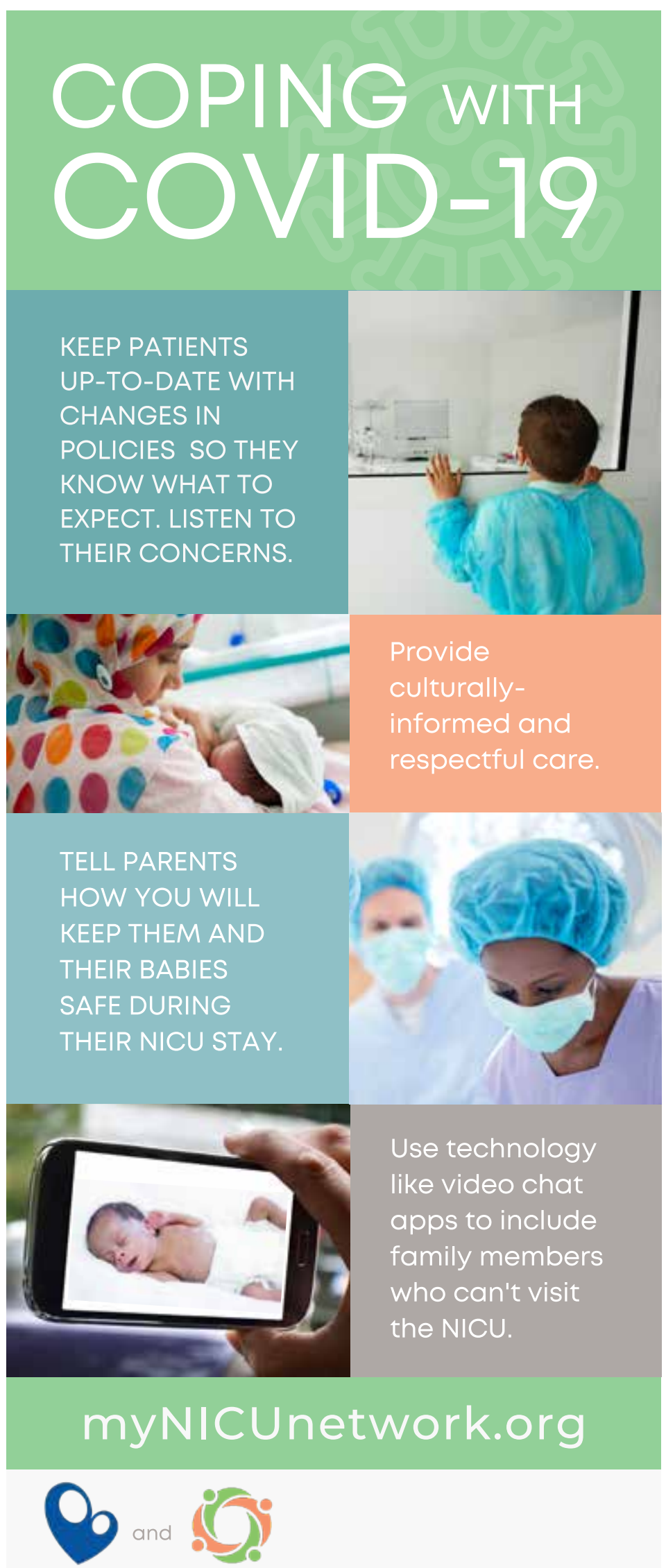

National Perinatal Association NICU Parent Network
My Perinatal Network and My NICU Network are products of a collaboration
between NPA and NPN. 